\title{
Ferroptosis is involved in the anti-tumor effect of lycorine in renal cell carcinoma cells
}

\author{
YANG DU $^{1 *}$, HONG-CHAO ZHAO ${ }^{1 *}$, HENG-CHENG ZHU ${ }^{1}$, \\ YAO JIN ${ }^{2}$ and LEI WANG ${ }^{1}$ \\ ${ }^{1}$ Department of Urology, Renmin Hospital of Wuhan University; ${ }^{2}$ Department of Surgery, Tongji Medical \\ College, Huazhong University of Science and Technology, Wuhan, Hubei 430000, P.R. China
}

Received April 9, 2021; Accepted July 14, 2021

DOI: $10.3892 / 01.2021 .13042$

\begin{abstract}
Renal cell carcinoma (RCC) is a most common malignant tumor in the genitourinary system. Studies have shown that Lycorine has promising anticancer activities with minor side effects. However, the effect of lycorine on the proliferation of RCC cells and its underlying anti-tumor mechanism have not yet been fully elucidated. The human renal cancer cell lines 786-O, A498 and Caki-1 were cultured and treated with different concentrations of lycorine or ferrostatin-1, a ferroptosis inhibitor. Cell viability and colony formation assays were used to measure cell proliferation. The 5-, 12- and 15-HETE hydroxyeicosatetraenoic acid (HETE) and MDA levels, as well as the reduced to oxidized glutathione (GHS/GSSG) ratio, were analyzed. Western blot analysis was used to detect the expression of glutathione peroxidase 4 (GPX4) and acyl-CoA synthetase long chain family member 4 (ACSL4), which are key markers of ferroptosis. Transmission electron microscopy was used to observe the morphological features associated with ferroptosis. Lycorine was found to inhibit the proliferation of RCC cells. After lycorine treatment,
\end{abstract}

Correspondence to: Professor Heng-Cheng Zhu, Department of Urology, Renmin Hospital of Wuhan University, 99 Zhang Zhi Dong Road, Wuhan, Hubei 430000, P.R. China

E-mail: phoenixneo@126.com

Professor Yao Jin, Department of Surgery, Tongji Medical College, Huazhong University of Science and Technology, 1095 Jiefang Road, Wuhan, Hubei 430000, P.R. China

E-mail: jinyao1024@hust.edu.cn

*Contributed equally

Abbreviations: RCC, renal cell carcinoma; HETE, hydroxyeicosatetraenoic acid; GSH, reduced glutathione; GSSG, oxidized glutathione; MDA, malondialdehyde; GPX4, glutathione peroxidase 4; ACSL4, acyl-CoA synthetase long chain family member 4

Key words: lycorine, renal cell carcinoma, ferroptosis, proliferation, ferrostatin-1 the expression levels of GPX4 in RCC cells decreased, whereas those of ACSL4 increased. Lycorine induced the expression of 5-HETE, 12-HETE, 15-HETE and MDA in RCC cells, and reduced the GSH/GSSG ratio. In addition, ferrostatin-1 could prevent lycorine-induced ferroptosis in RCC cells.

\section{Introduction}

Renal cell carcinoma (RCC; kidney cancer) is the third most common malignant tumor in the genitourinary system worldwide (1). There are $\sim 338,000$ new cases worldwide each year $(2,3)$. Despite advancements in the medical treatment for RCC in recent decades, including the development anti-angiogenic agents, receptor targeted therapy and immune checkpoint inhibition, the median overall survival rate for patients is still not satisfactory (4). There is evidence that the 5-year survival rate for patients with distant metastases is only $10 \%$ (5). Due to its high levels of resistance to chemotherapy and radiotherapy, radical surgery is still the only therapy for localized renal cell cancer (6). Therefore, it is urgent to develop new treatment targets and effective treatment methods.

Lycorine is a natural compound of pyrrolo[de]phenanthridine ring-type alkaloid extracted from the Amaryllidaceae genera (7). This compound has various biological effects including anti-virus (8), anti-malaria (9), anti-inflammation and anti-tumor effects, with mild side effects $(10,11)$. Although the potential targets and mechanisms of action of lycorine are still unclear, it is widely described as a candidate anti-tumor drug, due to its high anti-tumor activity (12). Previous studies have shown that lycorine has a selective cytotoxic effect on various cancer types (13-16). Moreover, a previous study reported that lycorine can target MEK2, induce autophagy-associated apoptosis, and enhance vemurafenib activity in colorectal cancer. However, hardly any research focused on its function in RCC (17).

Ferroptosis is a form of programmed cell death identified in recent years (18). The main morphological changes associated with ferroptosis include the shrinkage of mitochondria and mitochondrial ridge reduction (18). Ferroptosis can be induced by some specific small molecule compounds (such as erastin) and some common clinical drugs (such as sorafenib and artemisinin) $(19,20)$. The formation of ferroptosis is 
mainly ascribed to initial lipid peroxidation without the activation of caspases and the release of reactive oxygen species (ROS) in cells, which is closely related to the increase of iron ions in cells (21). Therefore, the entire ferroptosis process can be impaired by the inhibitor of lipid peroxidation, such as ferrostatin-1 (22). Ferroptosis plays an important role in the occurrence of a variety of tumors, including RCC (23). Therefore, activating ferroptosis in tumors has great therapeutic potential, which will provide new insight into the clinical treatment of RCC. The present study aimed to investigate the association between Lycorine and ferroptosis on the proliferation of human renal cancer cells carcinoma (RCC) cells and its specific anticancer mechanism.

\section{Materials and methods}

Cell culture and reagents. The human RCC cell lines, 786-O, A498 and Caki-1 cell lines were purchased from American Type Culture Collection, the human normal renal proximal tubular cell line HK-2 was purchased from Shanghai Bioleaf Biotech Co. Cells were cultured in DMEM medium (HyClone; Cytiva) supplemented with $10 \%$ fetal calf serum (Invitrogen; Thermo Fisher Scientific, Inc.) and penicillin/streptomycin (100 mg/ml; Thermo Fisher Scientific, Inc.) at $37^{\circ} \mathrm{C}$ in a $5 \% \mathrm{CO}_{2}$-humidified incubator. Lycorine (purity $>98 \%$ ) was purchased from MedChemExpress (cat. no. HY-N0288). A $50 \mathrm{mM}$ stock solution was prepared in dimethyl sulfoxide (DMSO; Sigma-Aldrich; Merck KGaA), stored at $-20^{\circ} \mathrm{C}$ and diluted as needed in cell culture medium.

Cell viability assay. Cell viability was determined using MTS assays. RCC or HK- 2 cells $\left(5 \times 10^{3}\right.$ cells/well) were treated with various concentrations of lycorine $(0,0.05,0.1,1,5,10,20$ and $50 \mu \mathrm{M}$ ) for $48 \mathrm{~h}$, unless otherwise stated. The Aqueous One Solution (Promega Corporation) was used for incubation for $120 \mathrm{~min}$ at $37^{\circ} \mathrm{C}$ according to the manufacturer's instructions, and the absorption of $490 \mathrm{~nm}$ was measured. Three independent experiments were carried out in triplicate.

Colony formation assay. Cells were seeded into a 6-well plate $\left(5 \times 10^{3}\right.$ cells/well $)$ and cultured for $24 \mathrm{~h}$. Cells were then treated with lycorine $(10 \mu \mathrm{M})$. On day 8 , colonies were fixed in $3.7 \%$ paraformaldehyde for $15 \mathrm{~min}$ at $37^{\circ} \mathrm{C}$, stained with $0.1 \%$ crystal violet for $30 \mathrm{~min}$ at $37^{\circ} \mathrm{C}$ and counted manually.

Transmission electron microscopy (TEM). Cells were washed with PBS, then fixed in $2.5 \%$ phosphate-buffered glutaraldehyde (Sigma-Aldrich; Merck KGaA) and 2\% osmium tetroxide for $3 \mathrm{~h}$ at $4^{\circ} \mathrm{C}$. The cells were then stained with $2 \%$ uranyl acetate at $37^{\circ} \mathrm{C}$ for $12 \mathrm{~h}$. After dehydration and polymerization, samples were cut into 70-90 nm ultrathin sections using an ultramicrotome (EMUC7, Leica Microsystems, Inc.). Cells were infiltrated in methanol/Lowicryl K4M (Wuhan Biofavor Biotech Services Co., Ltd.; in a series $2: 1,1: 1$ and $1: 2$ ) at $-30^{\circ} \mathrm{C}$ for $2 \mathrm{~h}$. Images were captured using a transmission electron microscope (FEI; Thermo Fisher Scientific, Inc.).

Mitochondrial membrane potential (MMP) assay. The mitochondrial membrane potential of the cells was investigated by tetramethylrhodamine methyl ester (TMRM) (AAT Bioquest, Inc.) staining. Briefly, cells were seeded in confocal dishes (1x $10^{5} /$ well) and cultured for $48 \mathrm{~h}$. The cells were washed twice with PBS, then incubated for $20 \mathrm{~min}$ at $37^{\circ} \mathrm{C}$ with $100 \mathrm{nM}$ TMRM to stain the mitochondria. TMRM intensity was determined using a confocal fluorescence microscope.

Western blot analysis. Total protein was extracted from cells using the M-PER ${ }^{\mathrm{TM}}$ Mammalian Protein Extraction reagent (Thermo Fisher Scientific, Inc.). The protein concentration was determined using BCA method. Protein samples were added to an equal volume of $2 \mathrm{X}$ SDS loading buffer and boiled for $5 \mathrm{~min}$. Proteins (30 $\mu \mathrm{g}$ per lane) were separated via SDS-PAGE on a $10 \%$ gel, then transferred to a polyvinylidene difluoride membrane (Beijing Zhongshan Jinqiao Biotechnology Co., Ltd.). The membrane was blocked with $5 \%$ skimmed milk (Beyotime Institute of Biotechnology) at room temperature for $2 \mathrm{~h}$ and incubated with primary antibodies against GPX4 (1:3,000; cat. no. sc-166570; Santa Cruz Biotechnology, Inc.), ACSL4 (1:2,000; cat. no. sc-365230; Santa Cruz Biotechnology, Inc.) or anti- $\beta$-actin (1:500; cat. no. SA00001-9; ProteinTech Group, Inc.) overnight at $2-8^{\circ} \mathrm{C}$. Following primary antibody incubation, membranes were incubated with a horseradish peroxidase-conjugated secondary antibody (1:1,000; cat. no. SA00001-9; ProteinTech Group, Inc.) at room temperature for 3 h. Protein bands were observed using an Amersham ECL Prime Western Blotting Detection Reagent (GE Healthcare Life Sciences).

5-hydroxyeicosatetraenoic acid (HETE) assay. 5-HETE, a ferroptotic marker (24), was assessed using a 5-HETE ELISA kit (cat. no. CED739Ge; Wuhan USCN Business Co., Ltd.), according to the manufacturer's protocol.

12/15HETE assay. 12-HETE and 15-HETE are two ferroptotic markers (25). 12- or 15-HETE levels were determined using 12- or 15-HETE ELISA kits (cat. nos. ab133034 and ab133035; Abcam), according to the manufacturer's protocols. Each 96-well plate includes control, blank, standard and sample wells, each in duplicate. First, $100 \mu \mathrm{l}$ of the appropriate diluent and $50 \mu \mathrm{l}$ assay buffer were added to all wells except blank wells, and $100 \mu \mathrm{l}$ of the appropriate diluent was added to the standard wells. Subsequently, $50 \mu 1$ 12- or 15-HETE alkaline phosphatase conjugate was added to NSB, standard and sample wells and $50 \mu 1$ 12- or 15-HETE antibody was added to the B0, standard wells and sample wells. The plates were incubated for $2 \mathrm{~h}$ on a $56 \mathrm{x}$ g plate shaker at room temperature, the contents of the wells were then washed three times. Subsequently, $5 \mu 1$ 12/15-HETE alkaline phosphatase conjugate was added to all wells except blank wells and $200 \mu \mathrm{l}$ pNpp substrate solution was added to each well. Plates were incubated at $37^{\circ} \mathrm{C}$ for $3 \mathrm{~h}$ without shaking. The reaction was then stopped by adding $50 \mu \mathrm{l}$ of stop solution to each well. The absorbance was measured at $405 \mathrm{~nm}$ using a microplate reader, and the formula provided by the manufacturer was used to calculate the average net absorbance measurement for each well, corresponding to the 12- or 15-HETE levels.

Determination of GSH (reduced glutathione)/GSSG (oxidized glutathione) and malondialdehyde (MDA) levels. 

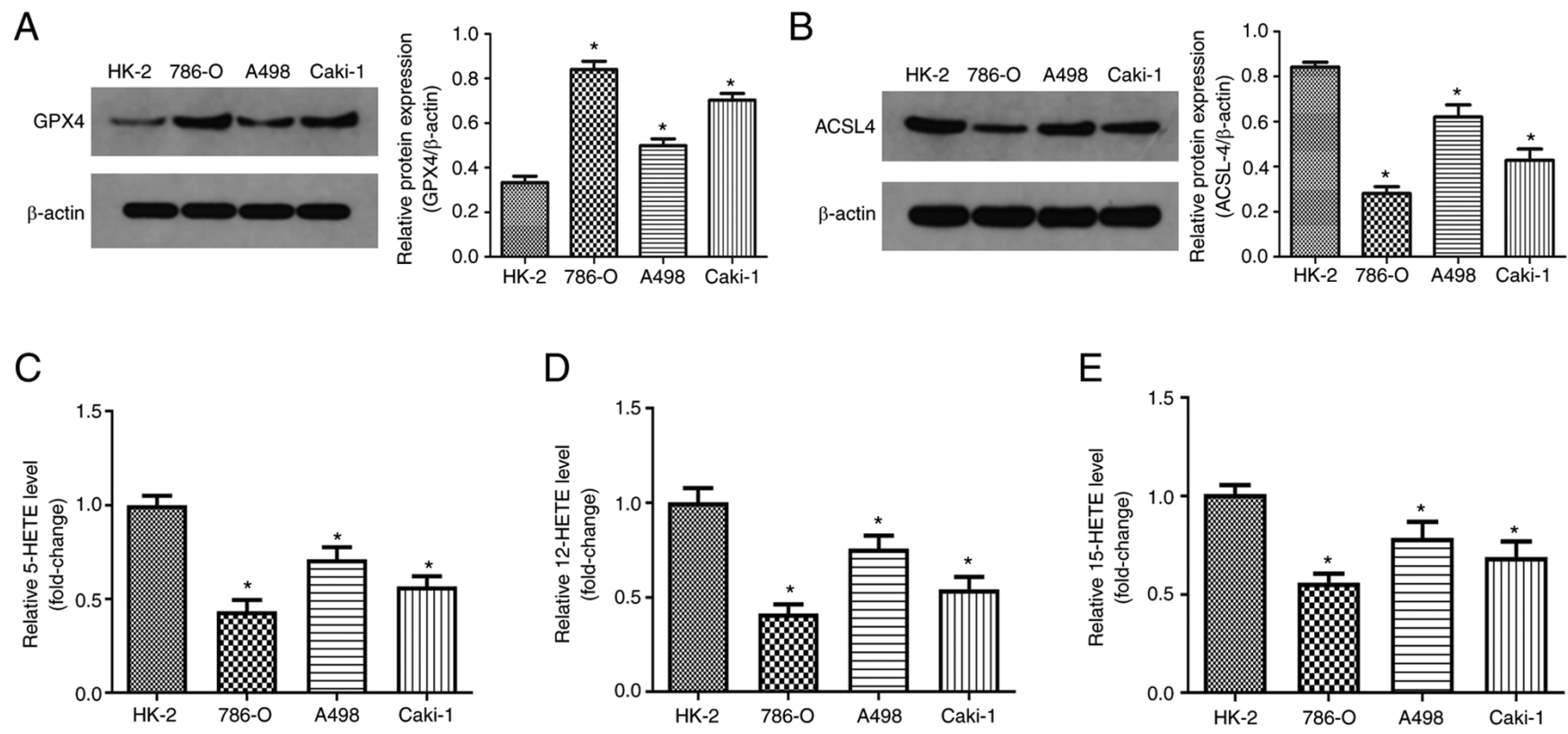

Figure 1. Ferroptosis levels in human RCC cells. (A) Representative western blots of GPX4 expression in human RCC cells, and quantification of the results. (B) Representative western blots of ACSL4 expression in normal renal proximal tubule cell line HK-2, and quantification of the results. Levels of (C) 5-HETE, (D) 12-HETE and (E) 15-HETE were detected in normal renal proximal tubule cell and RCC cells using commercial kits. "P<0.05 vs. control. GPX4, glutathione peroxidase 4; RCC, renal cell carcinoma; HETE, hydroxyeicosatetraenoic acid; 4; ACSL4, acyl-CoA synthetase long chain family member 4.

The levels of GSH and MDA in cell extracts were determined. The Reduced glutathione (GSH) was analyzed using the Reduced Glutathione Assay kit (Nanjing Jiancheng Bioengineering Institute; cat. no. A006), according to the manufacturer's instructions. Total glutathione/oxidized glutathione (GSH/GSSG) was determined using the Total Glutathione/Oxidized Glutathione Assay kit (Nanjing Jiancheng Bioengineering Institute; cat. no. A061). MDA levels were determined using a lipid peroxidation (MDA) test kit (Biovision, Inc.; cat. no. K739-100) according to the manufacturer's instructions.

Statistical analysis. All data are presented as the mean \pm SD. Statistical analysis was carried out using SPSS 22.0 software (IBM Corp.). The test methods were described in figure legends. Graphs were generated using Excel (Microsoft Corporation) or GraphPad Prism 5.00 for Windows (GraphPad Software, Inc.). All experiments were repeated at least three times. $\mathrm{P}<0.05$ was considered to indicate a statistically significant difference.

\section{Results}

Determination of ferroptosis levels in human RCC cells. Previous studies have indicated that RCC cells are highly sensitive to ferroptosis (26). Therefore, in the present study, ferroptosis levels were evaluated in human RCC cells. GPX4, 12-HETE and 15-HETE participate in ferritin deposition as lipid peroxides. These molecules have recently been recognized as direct markers of ferroptosis (24). Thus, GPX4, 5-HETE, 12-HETE, 15-HETE were used as indicators of ferroptosis in this study. A total of three typical malignant RCC cell lines (786-O, A498 and Caki-1) and a human normal renal proximal tubule cell line (HK-2) were used. Compared with the normal kidney cells HK-2, the protein levels of GPX4 significantly increased in RCC cells (Fig. 1A).

ACSL4 is considered to be the core regulatory gene of ferroptosis (27). The expression of ACSL4 in human RCC cells was significantly reduced compared with normal human renal cells (Fig. 1B). ELISA was used to detect 5-HETE, 12-HETE and 15-HETE levels. RCC cells displayed. significantly lower levels of 5-HETE, 12-HETE and 15-HETE than normal cells (Fig. 1C-E). The decrease of ACSL4 expression may be related to impaired ferroptosis in RCC cells. These results suggest that the levels of ferroptosis in human RCC cells are reduced.

Lycorine inhibits the proliferation of human RCC cells. The chemical structure of lycorine is shown in Fig. 2A. MTS and colony formation assays were used to study the anti-tumor activity of lycorine on RCC. MTS assays indicated that lycorine significantly inhibited the proliferation of RCC cells in a dose-dependent manner, with $\mathrm{IC}_{50}$ values of 5-10 $\mu \mathrm{M}$ (Fig. 2B). In addition, the inhibitory effect of lycorine on the three RCC cell lines was time-dependent (Fig. 2C). In the colony formation assay, $10 \mu \mathrm{M}$ lycorine had a significant inhibitory effect on the colony formation of the three RCC cell lines (Fig. 2D). These results indicated that lycorine effectively inhibited the proliferation of RCC cells.

Lycorine induces ferroptosis in human RCC cells by downregulating GPX4 and upregulating ACSL4. To determine whether the anti-tumor effect of lycorine was related to ferroptosis, the lipid peroxidation levels of lycorine were investigated in human RCC cells. Lipid peroxidation is a key step during ferroptosis. MDA is a natural by-product of lipid peroxidation and is commonly used as a marker of lipid peroxidation (28). After $24 \mathrm{~h}$ of $10 \mu \mathrm{M}$ lycorine treatment, the MDA levels and 
A

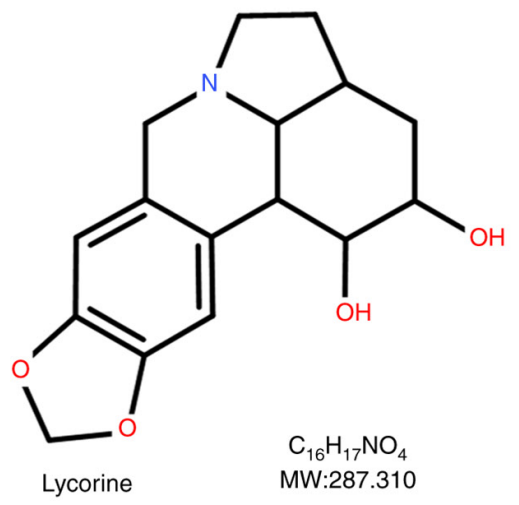

B

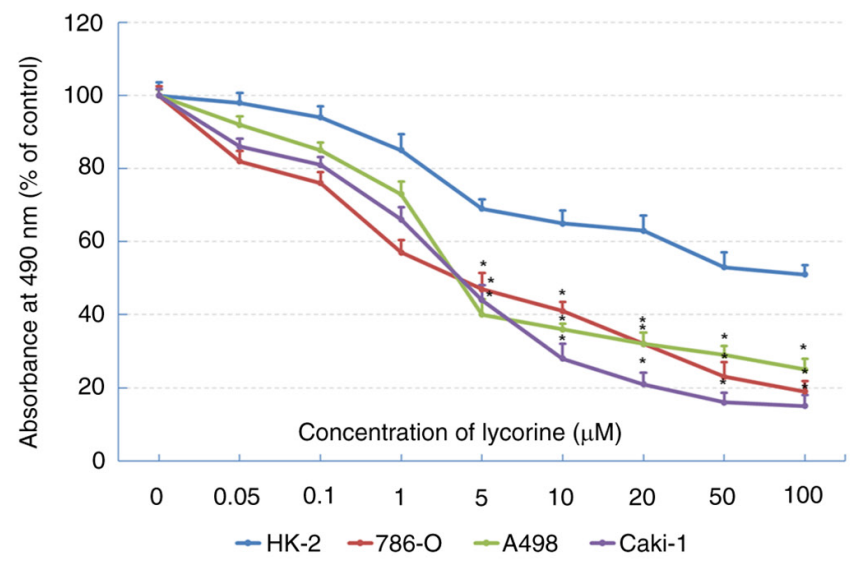

C

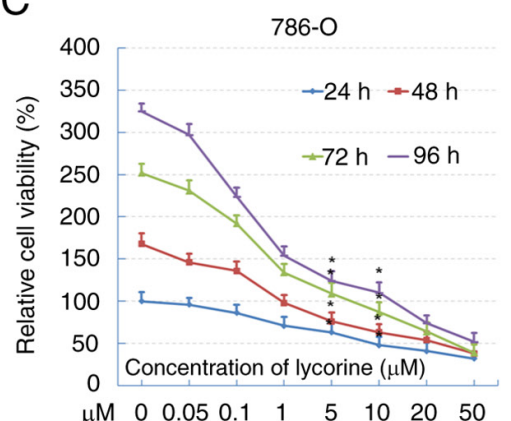

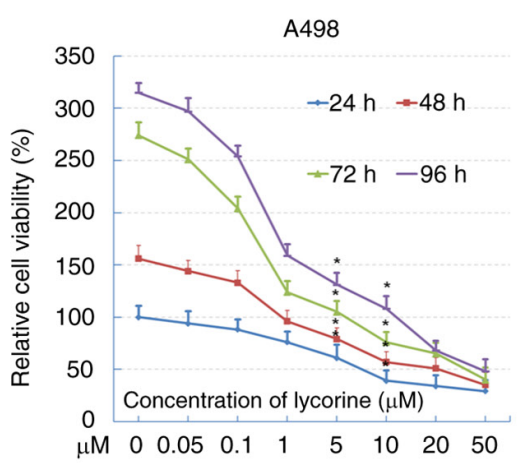

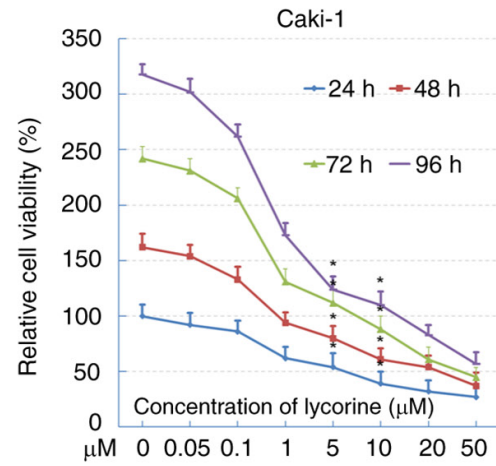

D

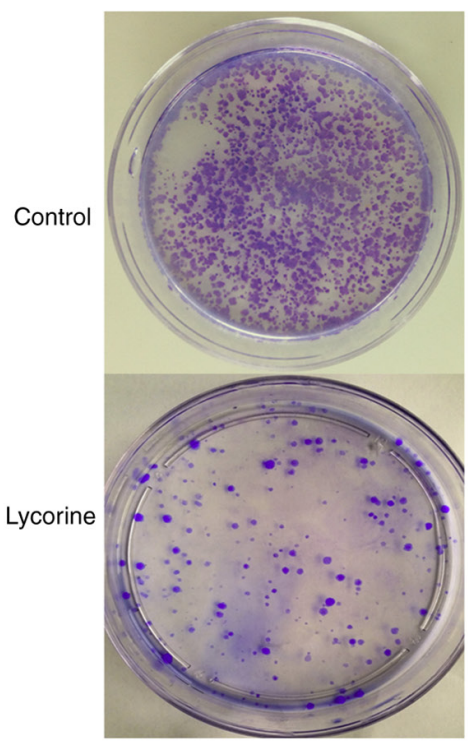

Lycorine: $10 \mu \mathrm{M}$
A498

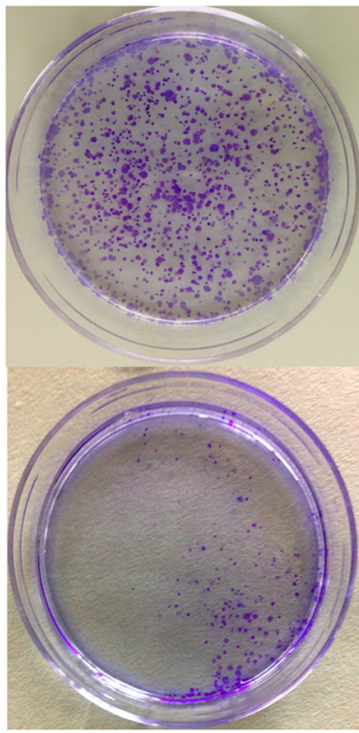

Caki-1

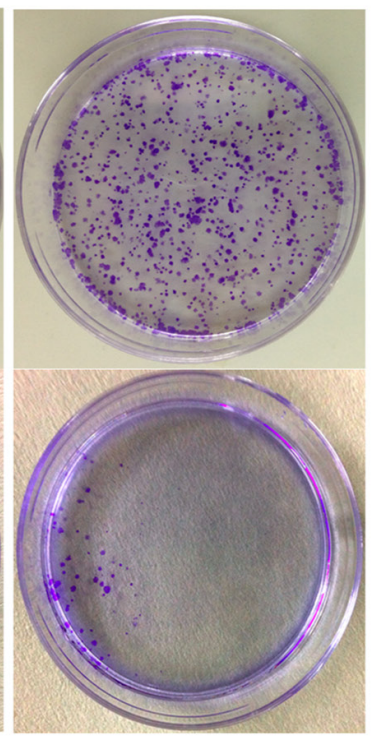

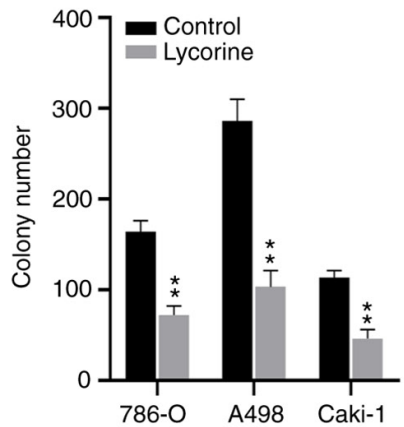

Figure 2. Effects of lycorine on proliferation and apoptosis of renal cell carcinoma cells. (A) Chemical structure of lycorine. (B) Cell viability in 786-O, A498 and Caki-1 and HK-2 cells treated with indicated concentrations of lycorine $(0-100 \mu \mathrm{M})$ for $48 \mathrm{~h} . \mathrm{P}=0.037$ between cell lines; $\mathrm{P}=0.029$ between doses; two-way ANOVA with Bonferroni correction. (C) Cell viability in 786-O, A498 and Caki-1 and HK-2 cells treated with lycorine with indicated concentrations $(0-50 \mu \mathrm{M})$ and for different incubation times $(24-96 \mathrm{~h})$ to test the time- and dose-dependent effects. $\mathrm{P}=0.006$ between time points; $\mathrm{P}=0.031$ between doses; two-way ANOVA with Bonferroni correction. (D) Colony formation assay in 786-O, A498 and Caki-1 treated with $10 \mu \mathrm{M}$ lycorine for $48 \mathrm{~h}$. The data are presented as the mean \pm SD from triplicate wells. ${ }^{~} \mathrm{P}<0.05,{ }^{* *} \mathrm{P}<0.01$ vs. control. MW, molecular weight.

ROS generation of the three RCC cell lines significantly increased compared with the control group (Fig. 3A-F).

GSH and GSSG are important cellular antioxidant systems that provide an inhibitory environment for the reduction of oxidized substances. GPX4 is an important regulator of ferroptosis. GPX4 deficiency causes a sharp increase in GSSG and a decrease in the GSH/GSSG ratio. Moreover, loss of GPX4 activity can lead to ferroptosis (29). In the present study, after 
A

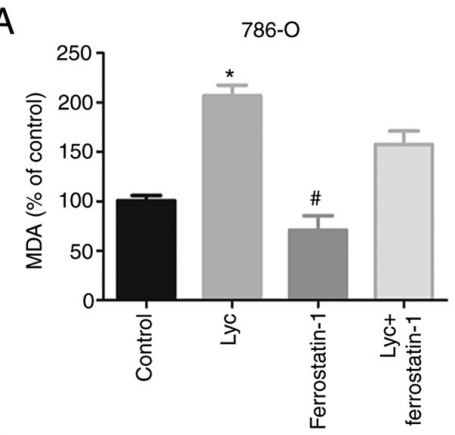

D

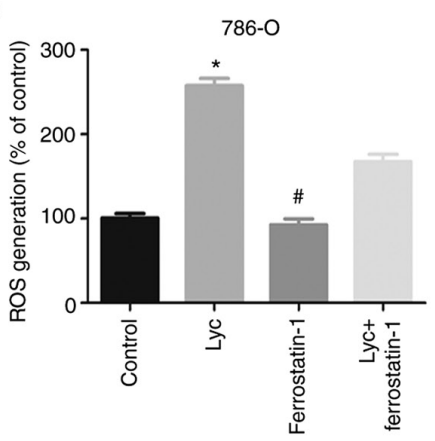

G

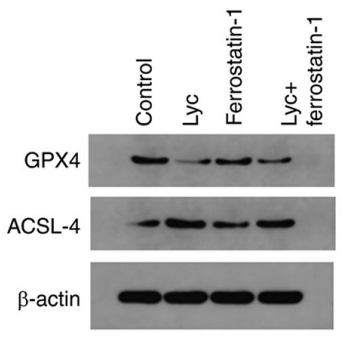

H

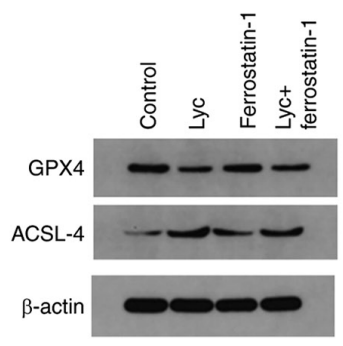

I

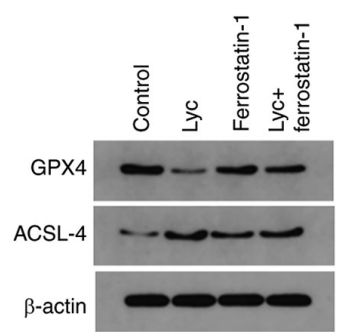

B

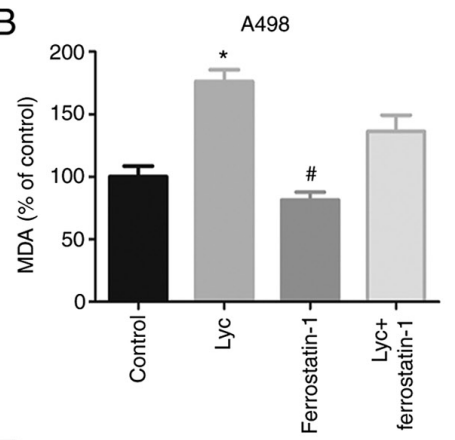

E
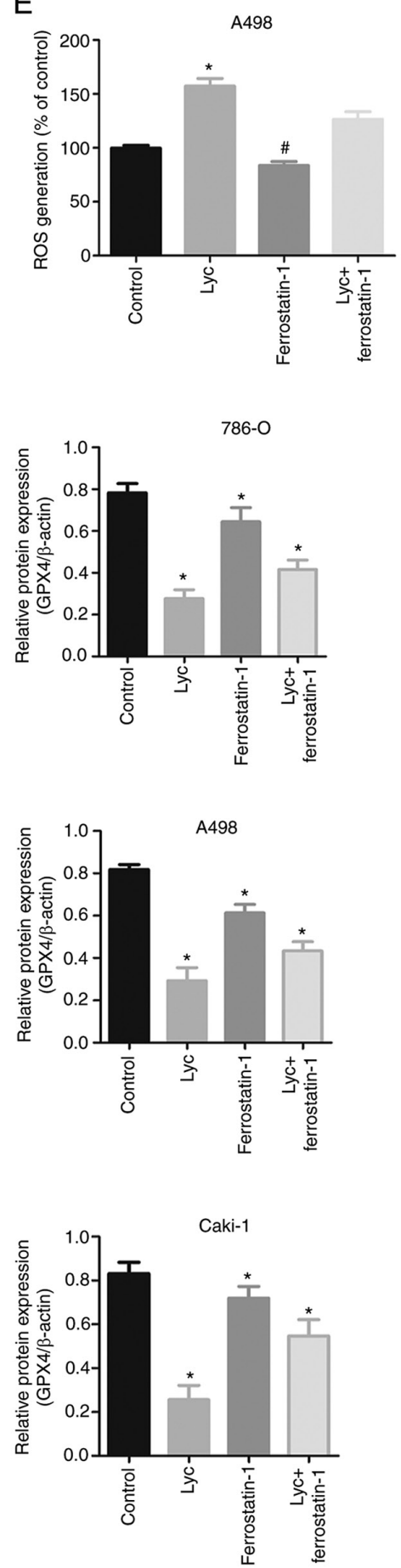

C

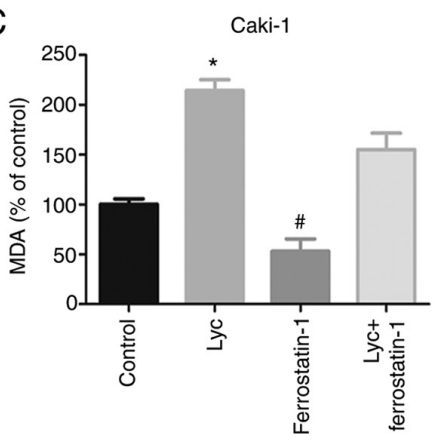

F
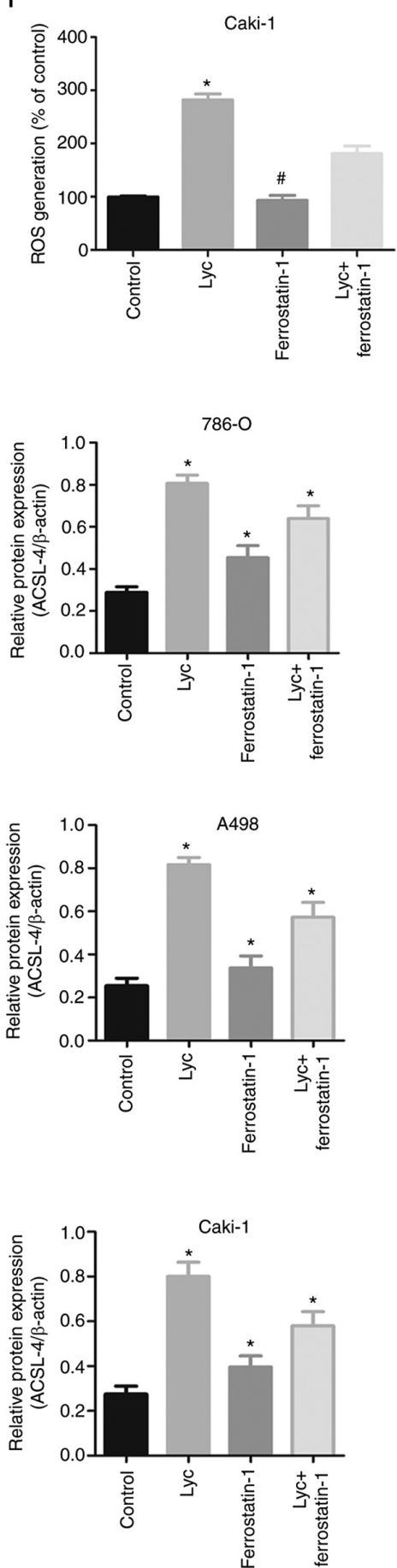

Figure 3. Ferroptosis inhibits the proliferation of RCC cells by regulating ferroptosis. 786-O, A498 and Caki-1 and HK-2 cells were treated with $10 \mu \mathrm{M}$ Lyc in the presence or absence of $1 \mu \mathrm{M}$ ferrostatin-1, a ferroptosis inhibitor. (A-C) MDA levels in RCC cells. (D-F) ROS generation in RCC cells. (G-I) Representative western blots of GPX4 and ACSL4 expression in RCC cells, with quantification of the results. ${ }^{\text {P }}<0.05$ vs. control; ${ }^{*} \mathrm{P}<0.05$ vs. Lyc. RCC, renal cell carcinoma; MDA, malondialdehyde; ROS, reactive oxygen species; ACSL4, acyl-CoA synthetase long chain family member 4; GPX4, glutathione peroxidase 4; Lyc, lycorine. 

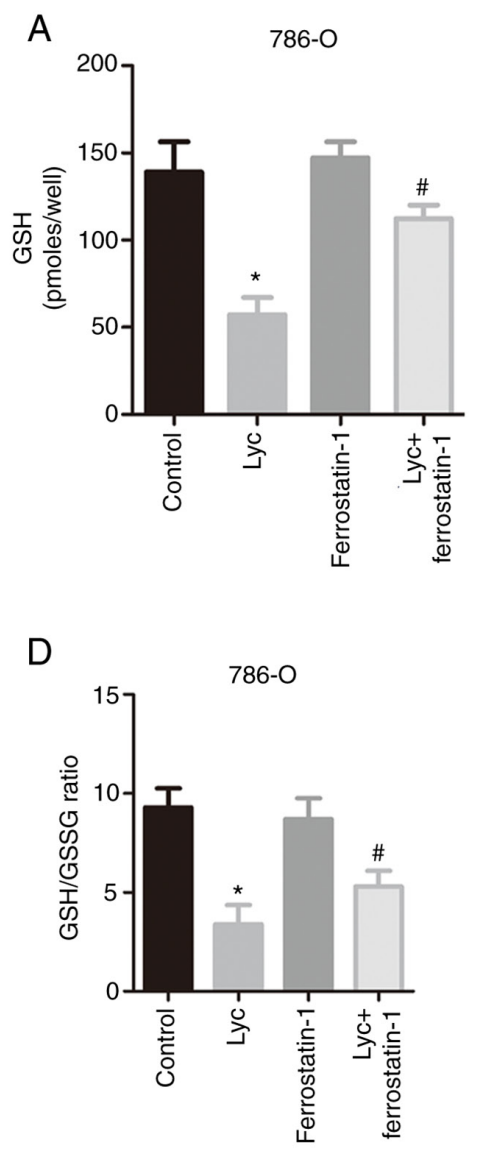

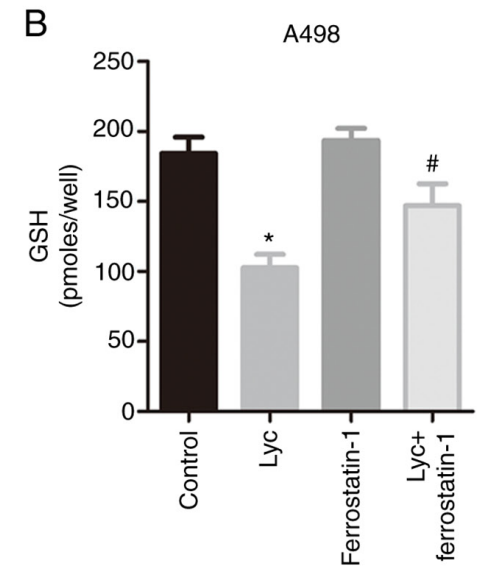

$\mathrm{E}$

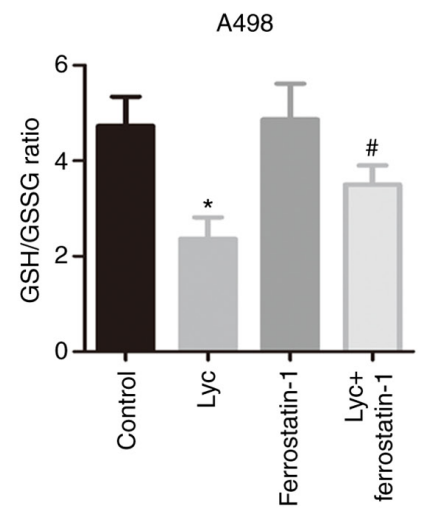

C

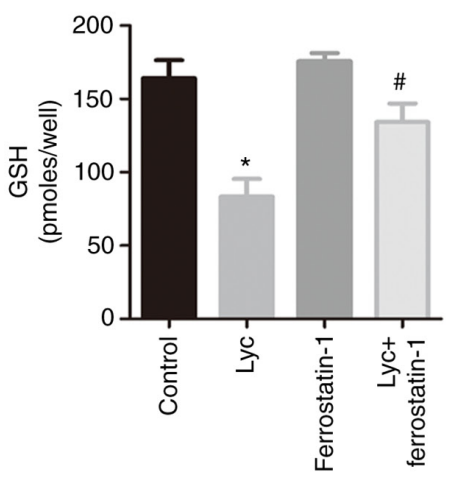

$\mathrm{F}$

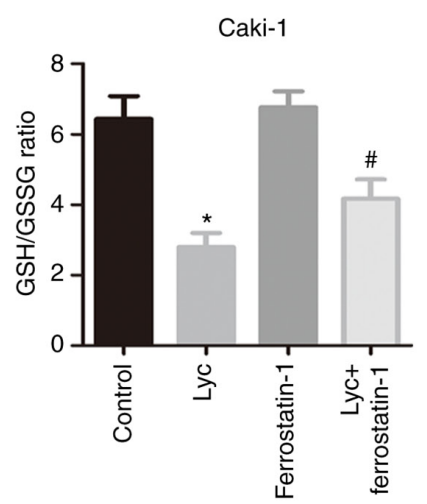

Figure 4. Lyc induces ferroptosis in RCC cells by modulating GSH levels. (A-C) GSH levels in RCC cells. (D-F) GSH/GSSG ratio in RCC cells. "P<0.05 vs. control; "P<0.05 vs. Lyc. RCC, renal cell carcinoma; GSH, reduced glutathione; GSSG, oxidized glutathione; Lyc, lycorine.

$24 \mathrm{~h}$ of treatment with lycorine, the expression of GPX4 in RCC cells was significantly reduced, while the expression of ACSL4 significantly increased (Fig. 3G-I). These results indicated that lycorine may induce ferroptosis in human RCC cells.

The anti-tumor effect of lycorine on RCC cells is blocked by ferroptosis inhibitors. Ferrostatin-1 is a specific inhibitor of ferroptosis (30), which can reduce the accumulation of intracellular ROS and cell death. It has been reported that ferrostatin-1 can inhibit ferroptosis by ferroptosis-inducers in vitro. In the present study, ferrostatin-1 significantly reduced the lycorine-induced decrease in GPX4 and increase in ACSL4 expression (Fig. 3G-I). Similarly, ferrostatin-1 reduced the increase in MDA levels induced by lycorine (Fig. 3A-C).

Furthermore, ferrostatin-1 abrogated the inhibitory effect on GSH induced by Lycorine (Fig. 4A, C and E) and also reversed the changes in GSH/GSSG ratio (Fig. 4B, D and F). In addition, the MTS assay was used to detect the effect of ferrostatin-1 on the viability of human RCC cells. The results showed that ferrostatin-1 reversed the inhibitory effect of lycorine on the viability of renal cancer cells (Fig. 5A-C). Moreover, TEM was used to observe the morphological features associated with ferroptosis. Clear mitochondrial shrinkage was observed, together with increased membrane density in the lycorine group compared with the control group. The morphological changes of mitochondria were also ameliorated in the lycorine + ferrostatin-1 group (Fig. 5D). TMRM staining was performed to evaluate the mitochondrial membrane potential following lycorine treatment. TMRM levels in the lycorine group was significantly reduced compared with the control group. However, ferrostatin-1 abrogated this suppressive effect (Fig. 5E). Thus, ferrostatin-1 abrogated the inhibitory effect of Lycorine on human RCC cells, suggesting that its anti-tumor effect on RCC cells can be attributed to its ferroptosis-inducing effect.

\section{Discussion}

Men with RCC are prone to peripheral invasion and distant metastasis, leading to death (31). Although great progress has been made in the treatment of RCC, treatment failure remains frequent (32). The main findings of the present study indicated that the anti-tumor activity of lycorine against RCC was moderate at physiologically achievable concentrations. These findings also demonstrated that RCC cells were prone to ferroptosis. Moreover, administration of lycorine inhibited the proliferation of RCC cells.

In recent years, Chinese herbal medicine have gradually attracted researchers' attention. Lycorine is one of the most commonly used Chinese herbal medicine. Lycorine has strong antiviral activity, including against severe acute respiratory syndrome associated coronavirus, poliovirus, enterovirus and hepatitis $\mathrm{C}$ virus (33-35). The anti-tumor activity of lycorine was first reported in 1976, when Jimenez et al (25) found that it inhibited protein synthesis in eukaryotic cells. Subsequently, an increasing number of studies identified lycorine as a potent tumor suppressor. For example, Wang et al (26) found that 

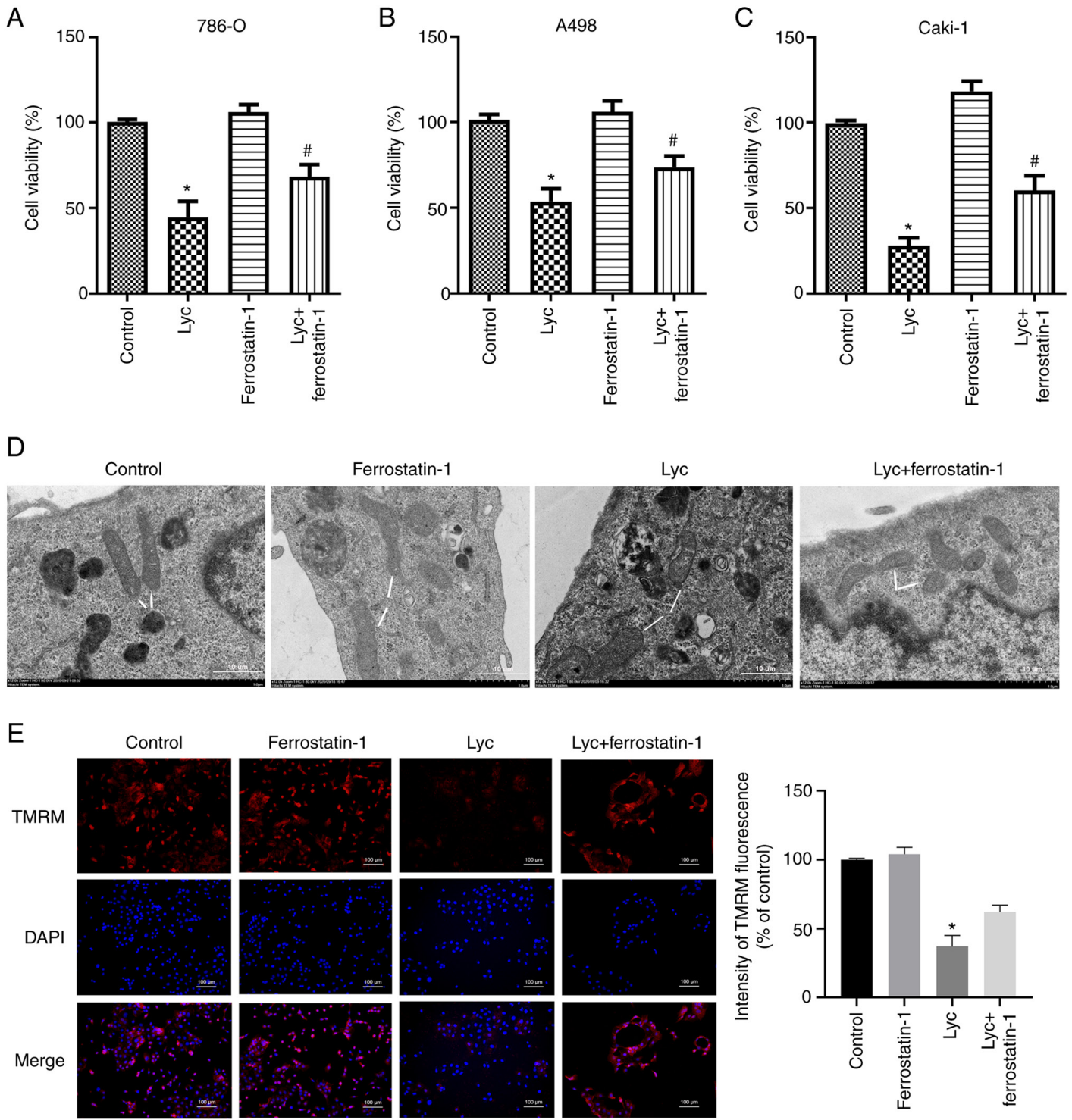

Figure 5. Ferrostatin-1 impairs the inhibitory effect of lycorine on RCC cells. (A-C) Proliferation of RCC cells was measured using a Cell Counting Kit-8 assay. (D) Ultrastructure of the renal tissue was captured by transmission electron microscopy at $24 \mathrm{~h}$. The white arrows indicate shrunken mitochondria. Scale bar, $10 \mu \mathrm{m}$. (E) Lyc reduces the mitochondrial membrane potential of RCC cells. The mitochondrial membrane potential in A498 cells following TMRM staining was observed using confocal fluorescence microscopy. Magnification, x200; Scale bar, $100 \mu \mathrm{m}$. ${ }^{*} \mathrm{P}<0.05$ vs. control; ${ }^{\text {* }} \mathrm{P}<0.05$ vs. Lyc. RCC, renal cell carcinoma; Lyc, lycorine; TMRM, tetramethylrhodamine methyl ester.

lycorine could target multiple myeloma stem cell-like cells through inhibition of the Wnt/ $\beta$-catenin pathway. Liu et al (27) have investigated the proapoptotic effect of lycorine in hepatoblastoma HepG2 cells. These studies suggested that lycorine has an inhibitory effect on different cancer types. However, few studies have focused on the effect of lycorine on RCC. Therefore, the present study explored the mechanism through which lycorine could inhibit the proliferation and promote the apoptosis of human RCC cells. In the present study, the proliferation of three RCC cell lines (786-O, A498 and Caki-1) was evaluated following treatment with lycorine. The results of MTS and colony formation assays suggested that lycorine had a significant inhibitory effect on the proliferation of human RCC cells. Its inhibitory effect was time- and dose-dependent.

Ferroptosis is an iron-dependent cell death progress. Recent studies have shown that Ferroptosis plays an important role in tumorigenesis (36). A large number of studies have shown that a variety of drugs can induce tumor cell ferroptosis at the protein and gene levels and trigger changes in GPX4 and ACSL4 expression $(37,38)$. Therefore, regulation of ferroptosis in tumor cells holds great potential for tumor treatment. In addition, the anti-tumor effect of lycorine is associated with ferroptosis, as evidenced by the inhibition of GPX4 expression and upregulation of ACSL4. The present study indicated that the expression of GPX4 in RCC cells was significantly reduced, while that of ACSL4 was increased following lycorine treatment. However, these effects were reversed following treatment with the ferroptosis inhibitor ferrostatin-1. These results suggest that the anti-tumor effect of lycorine on RCC may be related to the induction of ferroptosis. However, the present study lacks in vivo experiments.

In conclusion, the present study confirmed that lycorine could inhibit the proliferation in human RCC cells. The anti-tumor effect of lycorine was associated with the induction of ferroptosis. These findings indicated that lycorine could be 
used as a potential candidate anti-tumor drug, which will shed new light on the treatment against RCC.

\section{Acknowledgements}

Not applicable.

\section{Funding}

No funding was received.

\section{Availability of data and materials}

The datasets used and analyzed during the current study are available from the corresponding author on reasonable request.

\section{Authors' contributions}

YD was responsible conception and design. $\mathrm{HeCZ}$ and YJ contributed to the analysis of data and revised the manuscript. $\mathrm{HeCZ}$ and YJ agreed to be accountable for all aspects of the work in ensuring that questions related to the accuracy or integrity of any part of the work are appropriately investigated and resolved. $\mathrm{HeCZ}$ and $\mathrm{HoCZ}$ drafted and revised the manuscript, and confirm the authenticity of all the raw data. HoCZ and LW carried out the statistical analysis. All authors read and approved the final manuscript.

\section{Ethics approval and consent to participate}

Not applicable.

\section{Competing interests}

The authors declare that they have no competing interests.

\section{References}

1. Marques-Magalhães Â, Graça I, Miranda-Gonçalves V, Henrique R, Lopez M, Arimondo PB and Jerónimo C: Anti-neoplastic and demethylating activity of a newly synthetized flavanone-derived compound in renal cell carcinoma cell lines. Biomed Pharmacother 141: 111681, 2021.

2. Medina-Rico M, Ramos HL, Lobo M, Romo J and Prada JG: Epidemiology of renal cancer in developing countries: Review of the literature. Can Urol Assoc J 12: E154-E162, 2018.

3. Siegel RL, Miller KD and Jemal A: Cancer statistics, 2020. CA Cancer J Clin 70: 7-30, 2020.

4. Hsieh JJ, Purdue MP, Signoretti S, Swanton C, Albiges L, Schmidinger M, Heng DY, Larkin J and Ficarra V: Renal cell carcinoma. Nat Rev Dis Primers 3: 17009, 2017.

5. Sathianathen NJ, Krishna S, Anderson JK, Weight CJ, Gupta S, Konety BR and Griffith TS: The current status of immunobased therapies for metastatic renal-cell carcinoma. Immunotargets Ther 6: 83-93, 2017.

6. Argiris A, Harrington KJ, Tahara M, Schulten J, Chomette P, Ferreira Castro A and Licitra L: Evidence-based treatment options in recurrent and/or metastatic squamous cell carcinoma of the head and neck. Front Oncol 7: 72, 2017.

7. Lamoral-Theys D, Andolfi A, Van Goietsenoven G, Cimmino A, Le Calvé B, Wauthoz N, Mégalizzi V, Gras T, Bruyère $\mathrm{C}$, Dubois $\mathrm{J}$, et al: Lycorine, the main phenanthridine Amaryllidaceae alkaloid, exhibits significant antitumor activity in cancer cells that display resistance to proapoptotic stimuli: An investigation of structure-activity relationship and mechanistic insight. J Med Chem 52: 6244-6256, 2009.
8. Hwang YC, Chu JJ, Yang PL, Chen W and Yates MV: Rapid identification of inhibitors that interfere with poliovirus replication using a cell-based assay. Antiviral Res 77: 232-236, 2008.

9. Bendaif H, Melhaoui A, Ramdani M,Elmsellem H, Douez C and El Ouadi Y: Antibacterial activity and virtual screening by molecular docking of lycorine from Pancratium foetidum Pom (Moroccan endemic Amaryllidaceae). Microb Pathog 115: 138-145, 2018.

10. Fürst R: Narciclasine-an Amaryllidaceae alkaloid with potent antitumor and anti-inflammatory properties. Planta Med 82: 1389-1394, 2016.

11. Cole ER, de Andrade JP, Filho JFA, SchmittEFP, Alves-Araújo A, Bastida J, Endringer DC, de S Borges W and Lacerda V: Cytotoxic and genotoxic activities of alkaloids from the bulbs of griffinia gardneriana and habranthus itaobinus (Amaryllidaceae). Anticancer Agents Med Chem 19: 707-717, 2019.

12. Gao L, Feng Y, Ge C, Xu X, Wang S, Li X, Zhang K, Wang C, Dai $\mathrm{F}$ and Xie S: Identification of molecular anti-metastasis mechanisms of lycorine in colorectal cancer by RNA-seq analysis. Phytomedicine 85: 153530, 2021.

13. Liu J, Hu WX, He LF, Ye M and Li Y: Effects of lycorine on HL-60 cells via arresting cell cycle and inducing apoptosis. FEBS Lett 578: 245-250, 2004.

14. Li L, Dai HJ, Ye M, Wang SL, Xiao XJ, Zheng J, Chen HY, Luo YH and Liu J: Lycorine induces cell-cycle arrest in the G0/G1 phase in K562 cells via HDAC inhibition. Cancer Cell Int 12: 49, 2012.

15. Roy M, Liang L, Xiao X, Peng Y, Luo Y, Zhou W, Zhang J, Qiu L, Zhang S, Liu F, et al: Lycorine downregulates HMGB1 to inhibit autophagy and enhances bortezomib activity in multiple myeloma. Theranostics 6: 2209-2224, 2016.

16. Hu M, Peng S, He Y, Qin M, Cong X, Xing Y, Liu M and Yi Z: Lycorine is a novel inhibitor of the growth and metastasis of hormone-refractory prostate cancer. Oncotarget 6: 15348-15361, 2015.

17. Hu M, Yu Z, Mei P, Li J, Luo D, Zhang H, Zhou M, Liang F and Chen R: Lycorine induces autophagy-associated apoptosis by targeting MEK2 and enhances vemurafenib activity in colorectal cancer. Aging (Albany NY) 12: 138-155, 2020.

18. Cheng J, Fan YQ, Liu BH, Zhou H, Wang JM and Chen QX: ACSL4 suppresses glioma cells proliferation via activating ferroptosis. Oncol Rep 43: 147-158, 2020.

19. Ooko E, Saeed ME, Kadioglu O, Sarvi S, Colak M, Elmasaoudi K, Janah R, Greten HJ and Efferth T: Artemisinin derivatives induce iron-dependent cell death (ferroptosis) in tumor cells. Phytomedicine 22: 1045-1054, 2015.

20. Houessinon A, François C, Sauzay C, Louandre C, Mongelard G, Godin C, Bodeau S, Takahashi S, Saidak Z, Gutierrez L, et al: Metallothionein-1 as a biomarker of altered redox metabolism in hepatocellular carcinoma cells exposed to sorafenib. Mol Cancer 15: 38, 2016.

21. Qin Y, Qiao Y, Wang D, Tang C and Yan G: Ferritinophagy and ferroptosis in cardiovascular disease: Mechanisms and potential applications. Biomed Pharmacother 141: 111872, Jul 7, 2021 (Epub ahead of print).

22. Wu X, Yang Y, Xiang L and Zhang C: The fate of melanocyte: Mechanisms of cell death in vitiligo. Pigment Cell Melanoma Res 34: 256-267, 2021.

23. Yu H, Guo P, Xie X, Wang Y and Chen G: Ferroptosis, a new form of cell death, and its relationships with tumourous diseases. J Cell Mol Med 21: 648-657, 2017.

24. Yuan H, Li X, Zhang X, Kang R and Tang D: Identification of ACSL 4 as a biomarker and contributor of ferroptosis. Biochem Biophys Res Commun 478: 1338-1343, 2016.

25. Jimenez A, Santos A, Alonso G and Vazquez D: Inhibitors of protein synthesis in eukarytic cells. Comparative effects of some amaryllidaceae alkaloids. Biochim Biophys Acta 425: 342-348, 1976.

26. Wang H, Gong Y, Liang L, Xiao L, Yi H, Ye M, Roy M, Xia J, Zhou W, Yang C, et al: Lycorine targets multiple myeloma stem cell-like cells by inhibition of Wnt/beta-catenin pathway. Br J Haematol 189, 1151-1164, 2020.

27. Liu J, Kang R and Tang D: Signaling pathways and defense mechanisms of ferroptosis. FEBS J: Jun 6, 2021 (Epub ahead of print). doi: 10.1111/febs.16059.

28. Ni J, Chen K, Zhang J and Zhang X: Inhibition of GPX4 or mTOR overcomes resistance to lapatinib via promoting ferroptosis in NSCLC cells. Biochem Biophys Res Commun 567: 154-160, 2021.

29. Yang J, Ma S, Xu R, Wei Y, Zhang J, Zuo T, Wang Z, Deng H, Yang $\mathrm{N}$ and Shen Q: Smart biomimetic metal organic frameworks based on ROS-ferroptosis-glycolysis regulation for enhanced tumor chemo-immunotherapy. J Control Release 334: 21-33, 2021. 
30. Wenzel SE, Tyurina YY, Zhao J, St Croix CM, Dar HH, Mao G, Tyurin VA, Anthonymuthu TS, Kapralov AA, Amoscato AA, et al: PEBP1 wardens ferroptosis by enabling lipoxygenase generation of lipid death signals. Cell 171: 628-641.e26, 2017.

31. Chatzizacharias NA, Rosich-Medina A, Dajani K, Harper S, Huguet E, Liau SS, Praseedom RK and Jah A: Surgical management of hepato-pancreatic metastasis from renal cell carcinoma. World J Gastrointest Oncol 9: 70-77, 2017.

32. Ko JJ, Xie W, Kroeger N, Lee JL, Rini BI, Knox JJ, Bjarnason GA, Srinivas S, Pal SK, Yuasa T, et al: The international metastatic renal cell carcinoma database consortium model as a prognostic tool in patients with metastatic renal cell carcinoma previously treated with first-line targeted therapy: A population-based study. Lancet Oncol 16: 293-300, 2015.

33. Roy M, Liang L, Xiao X, Feng P, Ye M and Liu J: Lycorine: A prospective natural lead for anticancer drug discovery. Biomed Pharmacother 107: 615-624, 2018.

34. Chen D, Cai J, Yin J, Jiang J, Jing C, Zhu Y, Cheng J, Di Y, Zhang Y, Cao M, et al: Lycorine-derived phenanthridine downregulators of host $\mathrm{Hsc70}$ as potential hepatitis $\mathrm{C}$ virus inhibitors. Future Med Chem 7: 561-570, 2015.
35. Li SY, Chen C, Zhang HQ, Guo HY, Wang H, Wang L, Zhang X, Hua SN, Yu J, Xiao PG, et al: Identification of natural compounds with antiviral activities against SARS-associated coronavirus. Antiviral Res 67: 18-23, 2005.

36. Guo J, Xu B, Han Q, Zhou H, Xia Y, Gong C, Dai X, Li Z and Wu G: Ferroptosis: A novel anti-tumor action for cisplatin. Cancer Res Treat 50: 445-460, 2018.

37. Belavgeni A, Bornstein SR and Linkermann A: Prominin-2 suppresses ferroptosis sensitivity. Dev Cell 51: 548-549, 2019.

38. Liu N, Lin $X$ and Huang C: Activation of the reverse transsulfuration pathway through NRF2/CBS confers erastin-induced ferroptosis resistance. Br J Cancer 122: 279-292, 2020.

(c) (i) () This work is licensed under a Creative Commons Attribution-NonCommercial-NoDerivatives 4.0 International (CC BY-NC-ND 4.0) License. 